\title{
Variables salutogénicas y patogénicas, imagen corporal y calidad de vida relacionada con la salud en pacientes con cáncer de mama
}

\author{
Salutogenic and pathogenic variables, body image, \\ and health-related quality of life in breast cancer patients
}

\author{
Shirley Franco ${ }^{1}$, María Alejandra Zuluagal, Stefano Vinaccial, \\ Rosangela Raleigh ${ }^{1}$ y Gustavo Martínez ${ }^{2}$
}

Citación: Franco, S., Zuluaga, M.A., Vinaccia, S., Raleigh, R. y Martínez, G. (2019). Variables salutogénicas y patogénicas, imagen corporal y calidad de vida relacionada con la salud en pacientes con cáncer de mama. Psicología y Salud, 29(2), 225-235.

\section{RESUMEN}

\begin{abstract}
La presente investigación tuvo el objetivo de evaluar la relación de variables salutogénicas (resiliencia y esperanza), patogénicas (ansiedad y depresión), calidad de vida relacionada con la salud e imagen corporal en 50 mujeres con edades comprendidas entre 24 y 70 años, diagnosticadas con cáncer de mama, atendidas en el Instituto Médico de Alta Tecnología, en Montería, Colombia. Se utilizó una batería compuesta por un cuestionario de datos sociodemográficos, las escalas de Imagen Corporal, de Ansiedad y Depresión Hospitalaria y de Resiliencia de Connor-Davidson, el Índice Herth de Esperanza y el Cuestionario de Salud. Los resultados indican que las pacientes mostraron niveles no significativos de ansiedad y depresión, y óptimos de resiliencia y esperanza, así como una adecuada imagen corporal y una calidad de vida que osciló entre buena y bastante favorable. Por último, se encontraron relaciones que demuestran que las variables salutogénicas y patogénicas, en conjunto con la imagen corporal, se asocian con la calidad de vida relacionada con la salud de dichas pacientes.
\end{abstract}

Palabras clave: Cáncer de mama; Calidad de vida; Resiliencia psicológica; Depresión; Imagen corporal.

\begin{abstract}
The aim of this study was to assess the link between "salutogenic" (resilience and hope) and pathogenic variables (anxiety and depression) with health-related quality of life and body image in 50 patients aged 24 to 70 years, diagnosed with breast cancer, attending the "Instituto Médico de Alta Tecnología", in Montería, Colombia. A sociodemographic questionnaire and the following five instruments were applied: the Hospital Anxiety and Depression Scale, the Connor-Davidson Resilience Scale, the Herth Hope Index, the Short Form-36 Health Survey, and the Body Image Scale. The results showed no significant levels of anxiety and depression as well as optimal levels of resilience and hope, adequate body image, and quality of life ranging between good and very favorable. Finally, significant correlations between salutogenic and pathogenic variables, along with body image, are associated with health-related quality of life in these patients.
\end{abstract}

Key words: Breast cancer; Quality of life; Psychological resilience; Depression; Body image.

\footnotetext{
${ }^{1}$ Programa de Psicología, Universidad del Sinú, Carrera 1w-No.38-53, Montería, Córdoba, Colombia, tel. (0057)181-17-17, correos electrónicos: shirleyfranco@hotmail.com, zuluaga.maria@outlook.com, vinalpi47@hotmail.com y rosangela.raleigh@gmail.com.Artículo recibido el20de junio y aceptado el 26 de septiembre de 2018.

${ }^{2}$ Instituto Médico de Alta Tecnología Oncomédica, Carrera 6, Núm. 72-34, Monteria, Córdoba, Colombia, tel. (0057)785-43-44.
} 


\section{INTRODUCCIÓN}

$\mathrm{S}$ egún datos de la Organización Mundial de la Salud (2018), el cáncer es una de las principales causas de morbilidad y mortalidad en el mundo. En Latinoamérica, el de mama es el tipo de cáncer más frecuente, y casi 300 mil mujeres mueren anualmente por esta enfermedad (Organización Panamericana de la Salud, 2018). En Colombia, según cifras dadas por el Ministerio de Salud y Protección Social (2017a), fue la primera causa de muerte por cáncer en las mujeres, con un total de 2,381 decesos (Ministerio de Salud y Protección Social, 2017b). Además, las cifras de diagnósticos de este padecimiento aumentan anualmente. Se observan alrededor de 8,686 casos cada año, en su mayoría en las grandes ciudades del país, lo que hace que sea el principal tipo de cáncer y que se perfile como un grave problema de salud pública (Ministerio de Salud y Protección Social, 2017b).

\section{Depresión y ansiedad}

Recibir un diagnóstico de cáncer de mama es considerado como un evento vital estresante de alto impacto, al igual que el de todas las enfermedades oncológicas (Montalvo et al., 2016). Se estima que un tercio de los pacientes afrontan problemas psicológicos, como ansiedad y depresión (Fradelos et al., 2017). Al respecto, Reich, Lesur y Pedrizet (2008), en una revisión sistemática de la literatura sobre depresión, calidad de vida y cáncer de mama, encontraron que la depresión se diagnostica muchas veces erróneamente y que se le atiende de manera insuficiente en la población con ese tipo de cáncer. Los factores de riesgo de la depresión pueden estar asociados a la fatiga y a las actitudes cognitivas de desesperanza y resignación. Además, el deterioro de la imagen corporal debido a la quimioterapia, radioterapia, mastectomía y sus secuelas en la sexualidad genera tasas elevadas de trastornos del estado de ánimo.

\section{Imagen corporal}

En la cultura occidental, la imagen corporal parece estar basada en la juventud y el atractivo físico. Este énfasis puede eclipsar otros atributos perso- nales (Taub, Blinde y Greer, 1999). La importancia dada al cuerpo puede tener un efecto negativo en las personas con amputaciones, especialmente en la percepción del propio cuerpo (Sousa, Corredeira y Pereira, 2009). El estigma social y la ansiedad por tener una imagen corporal diferente tienden a estar asociados a un ajuste pobre en términos de una mayor restricción de actividad, y depresión y ansiedad generalizadas (Horgan y MacLachlan, 2004).

En España, Rincón, Pérez, Borda y Rodríguez (2012) analizaron en 72 pacientes si el nivel de satisfacción con el resultado estético, la autoestima o la imagen corporal diferían según el tipo de cirugía realizada (mastectomía unilateral o reconstrucción mamaria). En general, las pacientes reconstruidas mostraron una mayor satisfacción, más autoestima y un menor deterioro de su imagen corporal.

\section{Resiliencia}

Sin embargo, el predominio del efecto negativo que implican las condiciones desfavorables de salud o el deterioro funcional no excluyen la existencia de emociones positivas, como la alegría, la gratitud, el interés, la serenidad y el orgullo. De hecho, los efectos fisiológicos y psicológicos nocivos de los problemas médicos de tipo crónico pueden verse mitigados por esas emociones (Angner, Ghandhi, Williams-Purvis, Amante y Allison, 2013; Angner, Ray, Saag y Allison, 2009), que además sirven de marcos protectores para la salud física y mental en esas condiciones, tal como lo establece la teoría de la ampliación y construcción de las emociones positivas (Fredrickson, 2001; Garaigordobil, 2015). En efecto, las emociones positivas guardan una sólida relación con la capacidad de recuperación psicológica. Las personas que obtienen puntuaciones altas en resiliencia se caracterizan por una emotividad positiva y tienen un enfoque más activo acerca de la vida, son más curiosas y están más abiertas a nuevas experiencias (Tugade, Fredrickson y Feldman, 2004).

En Estados Unidos, Ong, Zautra y Reid (2010) llevaron a cabo una investigación para examinar el papel de la resiliencia psicológica y las emociones positivas en la experiencia cotidiana del catastrofismo ante el dolor. Una muestra de 95 
hombres y mujeres con dolor crónico completaron evaluaciones iniciales de neuroticismo y resiliencia psicológica, así como un cuestionario de datos demográficos, tras de lo cual redactaron diarios breves durante catorce días consecutivos sobre la intensidad del dolor, el catastrofismo y las emociones positivas y negativas experimentadas. Los análisis de modelos multinivel indicaron que, independientemente del nivel de neuroticismo, las emociones negativas, la intensidad del dolor, los ingresos económicos y la edad, las personas con una alta resiliencia informaron tener más emociones positivas y exhibieron un catastrofismo de dolor más bajo que aquéllas con baja resiliencia. Los análisis revelaron además que los individuos psicológicamente resilientes se recuperan de la catastrofización diaria ocasionada por el dolor a través de experiencias emocionales positivas.

\section{Esperanza}

Otra variable positiva que se ha estudiado frecuentemente en esta área es la esperanza, un importante recurso psicológico para personas con enfermedades crónicas que se considera esencial para la vida (Eliott y Oliver, 2009). La esperanza puede definirse como un estado de motivación positiva basado en tres componentes: objetivos a alcanzar, estrategias para alcanzar esos objetivos y motivación para hacerlo (Seligman y Csikszentmihalyi, 2000). En diferentes estudios se ha hallado evidencia de los efectos positivos que tiene la esperanza sobre la salud. En general, las personas con más esperanza tienen menos probabilidades de ser diagnosticadas con enfermedades del aparato respiratorio (Richman et al., 2005), muestran mejoras en la adhesión terapéutica y en sus estilos de vida mientras están en procesos de rehabilitación (Halding y Heggdal, 2012), y tienen una menor probabilidad de que reaparezcan en ellas los síntomas de ciertas enfermedades crónicas (Waynor, Gao, Dolce, Haytas y Reilly, 2012).

\section{Calidad de vida}

Tanto la resiliencia psicológica como la esperanza tienen relación con otras variables importantes en las pacientes con cáncer de mama, como la calidad de vida relacionada con la salud (CVRS en lo su- cesivo), la que se ve afectada no únicamente por las características de la enfermedad, sino también por diversos factores sociodemográficos, laborales, clínicos y psicosociales. Por ejemplo, Ristevska-Dimitrovska, Stefanovski, Smichkoska, Raleva y Dejanova (2015) evaluaron la calidad de vida y la resiliencia en 218 pacientes macedonias con cáncer de mama mediante los instrumentos EORTCQLQ Core 30 y EORTC QLQ-BR23 para la primera variable, y el CD-RISC 10 para la segunda. Sus resultados muestran que la resiliencia se asocia con diferentes dimensiones de la CVRS, pues todas las puntuaciones de las escalas funcionales del EORTC QLQ correlacionaron negativamente con ella.

En China, Zhang, Zhao, Cao y Ren (2017) aplicaron la escala de resiliencia CD-RISC 10, la escala MOS de apoyo social y el cuestionario de calidad de vida FACT-B a una muestra de 98 pacientes. Se encontró que las participantes con mayor apoyo social mostraron una mayor capacidad de recuperación y una mejor calidad de vida. El apoyo social desempeña, pues, un rol mediador en la relación entre la resiliencia y la calidad de vida.

En Colombia se han hecho estudios sobre la calidad de vida en general de pacientes con cáncer de mama (Pineda, Andrade y Montoya, 2013; Salas y Grisales, 2010), tanto en los diferentes estadios de la enfermedad (Aguirre, Núñez, Navarro y Cortés, 2017), como en su relación con el apoyo social (Martínez, 2018).

A partir de lo anterior, y ante la escasa investigación que hay en el país sobre la calidad de vida en mujeres con diagnóstico de cáncer de mama y su relación con otras variables psicológicas importantes, se propuso como objetivo del presente estudio evaluar la concordancia de las variables salutogénicas (resiliencia y esperanza) y patogénicas (ansiedad y depresión) con la CVRS y la imagen corporal en una muestra de pacientes con diagnóstico de cáncer de mama, residentes en la ciudad de Montería (Colombia).

\section{MÉTODO}

\section{Participantes}

El presente estudio se realizó con 50 mujeres mediante un muestreo no probabilístico por conve- 
niencia. Los criterios de inclusión fueron tener un diagnóstico de cáncer de mama, estar siendo atendidas en el Instituto Médico de Alta Tecnología en la referida ciudad y encontrarse en tratamiento durante la aplicación de los instrumentos.

\section{Instrumentos}

Escala Hospitalaria de Ansiedad y Depresión (HADS). Diseñada por Zigmond y Snaith (1983), la versión en español fue adaptada y validada en pacientes colombianos por Rico, Restrepo y Molina (2005). El coeficiente alfa de Cronbach original de 0.85 fue en el presente estudio de 0.67. Para la validez de constructo se empleó el análisis factorial exploratorio, que dio dos factores, tal como corresponde a las dos subdimensiones de la escala. Es este un cuestionario autoaplicado que consta de catorce ítems que se califican en una escala Likert con recorrido de 0 a 3. Está dividido en dos subescalas: ansiedad y depresión. Los ítems impares evalúan la primera y los pares la segunda. El rango de puntuación en cada subescala es de 0-21, donde a mayor puntaje, mayor ansiedad o depresión.

Connor-Davidson Resilience Scale (CD-RISC 10). En la versión reducida de diez ítems, esta escala, desarrollada por Campbell-Sills y Stein (2007) y adaptada al español por Notario et al. (2011), fue validada por Riveros, Bernal, Bohórquez, Vinaccia y Quiceno (2016) en enfermos crónicos colombianos. Tiene cinco opciones de respuesta tipo Likert en un rango que va de 0 a 4 puntos, y un coeficiente alfa de Cronbach de 0.82, que en el presente estudio fue de 0.84 . Se encontró una adecuada validez de constructo y concordancia con la estructura de la escala original. Las puntuaciones por debajo de 27 se consideran bajas, y altas por encima de 36.

Herth Hope Index (HHI). Es la versión reducida de The Herth Hope Scale (HHS), desarrollada por Herth (1991) y validada en español por Castilla, Urruti, Shimabukuro y Caycho (2014) en Perú. Consta de diez ítems que se evalúan en una escala Likert de cuatro opciones. La puntuación total máxima es de 40 puntos, y una mayor puntuación indica un mayor nivel de esperanza. La escala tiene una consistencia interna de 0.85 , aunque en este estudio fue de 0.81 .

Medical Outcomes Study-Short Form 36 (MOS$S F$ 36). Diseñado por Ware y Sherbourne, (1992), su adaptación al español fue hecha por Alonso, Prieto y Antó (1995) y Alonso et al. (1998), y validada para la población adulta colombiana por Lugo, García y Gómez (2006). Este cuestionario consta de 36 ítems que se responden asimismo en una escala Likert. Sus ítems se dividen en ocho dimensiones: función física, rol físico, dolor corporal, salud general, vitalidad, función social, rol emocional y salud mental. Además, se organiza en dos índices sumarios: uno para salud física y otro para salud mental. Su fiabilidad se encuentra entre 0.80 y 0.92 en sus diferentes dimensiones, pero en el presente estudio se ubicó entre $0.57 \mathrm{y}$ 0.89 . El punto de corte es de 50; por encima de este puntaje se considera que hay estados positivos de salud, y estados negativos por debajo de ese número.

Body Scale Inventory (BSI). La Escala de Imagen Corporal fue elaborada por Hopwood, Fletcher, Lee y Al Ghazal (2001), y Gómez, Bragado, Hernández y Sánchez (2015) la adaptaron al español. Está compuesta por diez ítems en formato Likert que va de 0 ("en absoluto") a 3 ("mucho"). La puntuación total se ubica en un rango de 0 a 30 puntos, en el que las puntuaciones de 0 representan la ausencia de síntoma, y las elevadas una tendencia a la alteración de la imagen corporal. El coeficiente alfa de Cronbach de la escala, de 0.93, fue en este estudio de 0.84 .

\section{Procedimiento}

Luego del aval otorgado por los comités éticos del Instituto Médico de Alta Tecnología (IMAT) y de la Universidad del Sinú, se llevó a cabo la selección de pacientes por conveniencia y según los criterios de inclusión establecidos. Así pues, a las pacientes que padecían cáncer de mamá y acudían a quimioterapia en las instalaciones del IMAT de la mencionada localidad se les informaron los objetivos de la investigación y se solicitó su consentimiento para participar. A fin de preservar su integridad, la confidencialidad de los datos y el 
respeto a su dignidad, todo lo anterior se hizo bajo las normas de la Declaración Universal de Principios Éticos para Psicólogos (International Union of Psychological Science, 2008), la Declaración de Helsinki de la AMM sobre los principios éticos para las investigaciones médicas en seres humanos (World Medical Organization, 2013) y el Código de Conducta y Principios Éticos para Psicólogos de la American Psychological Association (2017).

Una vez obtenido su consentimiento, se hizo el llenado de la ficha de datos sociodemográficos y se aplicó a las participantes la batería de pruebas compuesta por los cinco instrumentos citados (HADS, MOS-SF 36, HHI, CD-RISC 10 y BSI), lo que tuvo una duración aproximada de 30 minutos por cada paciente; luego se llevó a cabo la transcripción y el procesamiento de los datos utilizando el programa IBM SPSS, versión 23; por último, se realizó un análisis descriptivo de las variables sociodemográficas y de los puntajes de las pruebas y correlaciones, para lo cual se utilizó la prueba Shapiro-Wilk para determinar la normalidad de la distribución de las puntuaciones. De acuerdo a los resultados, se procedió a utilizar el coeficiente de correlación de Spearman.

\section{RESULTADOS}

La muestra estuvo compuesta por 50 pacientes de entre 24 y 70 años, con una edad media de 50.42 años y una desviación típica de 11.28. E1 tiempo mínimo transcurrido desde el diagnóstico fue de 2 meses y el máximo de 180. En la Tabla 1 se muestran los porcentajes y frecuencias de las características analizadas, esto es, el estadio de la enfermedad, las cirugías a que habían sido sometidas las participantes, su nivel educativo, ocupación y estado civil, y en la Tabla 2 las medidas de tendencia central (media y desviación típica) y los puntajes mínimos y máximos de cada uno de los instrumentos utilizados en el estudio.

En relación al HADS, los valores de las medias y las desviaciones típicas indican que, en general, la muestra no manifestaba síntomas clínicos de ansiedad o depresión, según los estándares de referencia de la escala en su versión colombiana (Rico et al., 2005), que indican puntos de corte de
8 y 9 para determinar la presencia de ansiedad y depresión hospitalarias, respectivamente.

Tabla 1. Características generales de la muestra estudiada de pacientes con cáncer.

\begin{tabular}{|c|c|c|}
\hline Caracterización & $\%$ & $\mathbf{N}$ \\
\hline \multicolumn{3}{|l|}{ Estadio de la enfermedad } \\
\hline I & 2 & 1 \\
\hline II & 58 & 29 \\
\hline III & 40 & 20 \\
\hline \multicolumn{3}{|l|}{ Cirugías } \\
\hline Cuadrantectomía & 22 & 11 \\
\hline Mastectomía simple & 10 & 5 \\
\hline Matectomía radical & 28 & 14 \\
\hline \multicolumn{3}{|l|}{ Nivel educativo } \\
\hline Primaria & 22 & 11 \\
\hline Secundaria & 28 & 14 \\
\hline Técnica & 24 & 12 \\
\hline Licenciatura & 16 & 8 \\
\hline Posgrado & 10 & 5 \\
\hline \multicolumn{3}{|l|}{ Ocupación } \\
\hline Amas de casa & 62 & 31 \\
\hline Empleadas & 24 & 12 \\
\hline Trabajadoras independientes & 8 & 4 \\
\hline Jubiladas & 4 & 2 \\
\hline Desempleadas & 2 & 1 \\
\hline \multicolumn{3}{|l|}{ Estado civil } \\
\hline Solteras & 10 & 5 \\
\hline Casadas & 28 & 14 \\
\hline Divorciadas o separadas & 30 & 15 \\
\hline Unión libre & 28 & 14 \\
\hline Viudas & 4 & 2 \\
\hline
\end{tabular}

Tabla 2. Estadísticas descriptivas de las variables bajo estudio.

\begin{tabular}{|l|r|r|r|r|}
\hline \multicolumn{1}{|c|}{ Instrumentos } & \multicolumn{1}{c|}{ M } & D.T & Mín. & Máx. \\
\hline HADS: Ansiedad & 4.26 & 3.09 & 0 & 12 \\
\hline HADS: Depresión & 2.80 & 2.61 & 0 & 12 \\
\hline HHI: Esperanza & 34.72 & 3.25 & 24 & 40 \\
\hline CD-RISC 10: Resiliencia & 32.88 & 5.54 & 17 & 40 \\
\hline BIS: Imagen corporal & 3.46 & 4.03 & 0 & 17 \\
\hline MOS-SF 36: Calidad de vida relacionada con la salud \\
\hline Función física & 91.40 & 16.32 & 10 & 88 \\
\hline Rol físico & 36.50 & 38.20 & 0 & 100 \\
\hline Dolor corporal & 70.28 & 26.95 & 0 & 100 \\
\hline
\end{tabular}

Continúa.. 


\begin{tabular}{|l|r|r|r|r|}
\hline Salud general & 74.64 & 17.55 & 37 & 100 \\
\hline Vitalidad & 71.80 & 17.13 & 35 & 100 \\
\hline Función social & 71.48 & 24.84 & 0 & 100 \\
\hline Rol emocional & 76.00 & 38.73 & 0 & 100 \\
\hline Salud mental & 75.28 & 18.26 & 24 & 100 \\
\hline Índice sumario físico & 65.66 & 17.34 & 28 & 97 \\
\hline Índice sumario mental & 73.82 & 17.51 & 35 & 98 \\
\hline
\end{tabular}

En cuanto a la resiliencia, las pacientes obtuvieron por lo común puntuaciones promedio según los puntos de corte señalados, y respecto a la esperanza, esos puntajes fueron superiores a 20 , que se espera sea la media en la escala Likert que se utiliza para responder el instrumento.

Con relación a la escala BIS, donde una mayor puntuación implica una mayor alteración de la imagen corporal, la media de 3.46 se ubica muy por debajo de los puntajes medios planteados por Hopwood et al. (2001) para pacientes de cáncer de mama, cuya media es de 8.07. Respecto al MOS-SF 36, que evalúa la CVRS, hubo puntajes por encima de 50 en todas las dimensiones, con excepción del rol físico, que fue de 36.50 (Tabla 3).

Tabla 3. Pruebas de normalidad para puntuaciones del BIS, HADS, CD-RISC 10 y SF-36.

\begin{tabular}{|l|c|r|c|r|}
\cline { 2 - 5 } \multicolumn{1}{c|}{} & \multicolumn{2}{c|}{ Kolmogorov-Smirnov } & \multicolumn{2}{c|}{ Shapiro-Wilk } \\
\cline { 2 - 5 } \multicolumn{1}{c|}{ Estadístico } & \multicolumn{1}{c|}{ Sig. } & Estadístico & \multicolumn{1}{c|}{ Sig. } \\
\hline Función física & 0.451 & 0 & 0.255 & 0 \\
\hline Rol físico & 0.238 & 0 & 0.809 & 0 \\
\hline Dolor corporal & 0.165 & 0.002 & 0.899 & 0 \\
\hline Salud general & 0.142 & 0.013 & 0.940 & 0.013 \\
\hline Vitalidad & 0.134 & 0.025 & 0.963 & 0.115 \\
\hline Función social & 0.147 & 0.009 & 0.910 & 0.001 \\
\hline Rol emocional & 0.412 & 0 & 0.629 & 0 \\
\hline Salud mental & 0.143 & 0.012 & 0.939 & 0.013 \\
\hline $\begin{array}{l}\text { Índice sumario } \\
\text { de salud física }\end{array}$ & 0.105 & $.200 *$ & 0.972 & 0.287 \\
\hline $\begin{array}{l}\text { Índice sumario } \\
\text { de salud mental }\end{array}$ & 0.138 & 0.019 & 0.921 & 0.003 \\
\hline Dolor corporal & 0.208 & 0 & 0.881 & 0 \\
\hline Total CD-RIsC 10 & 0.100 & $.200 *$ & 0.932 & 0.007 \\
\hline Total HHI & 0.119 & 0.076 & 0.942 & 0.017 \\
\hline Total BIS & 0.229 & 0 & 0.820 & 0 \\
\hline HADS ansiedad & 0.113 & 0.129 & 0.947 & 0.026 \\
\hline HADS depresión & 0.170 & 0.001 & 0.866 & 0 \\
\hline
\end{tabular}

* Límite inferior de la significación verdadera.

Para la evaluación de la normalidad en la distribución de las puntuaciones se tomaron en cuenta los resultados de la prueba Shapiro-Wilk debido al tamaño de la muestra $(n=50)$. De acuerdo a estos, en todos los casos (exceptuando las dimensiones de vitalidad y el sumario de salud física) se rechazó el supuesto de normalidad y se concluyó que los valores no se ajustaban a una distribución de ese tipo, por lo que se procedió a utilizar el coeficiente de correlación de Spearman para evaluar las relaciones entre variables, lo que se muestra en la Tabla 4.
El análisis de las correlaciones obtenidas al llevar a cabo las correlaciones de Spearman entre edad, tiempo de diagnóstico y las variables que evalúa el MOS-SF 36 evidencia que todas ellas fueron negativas, lo que indica que a mayor tiempo de diagnóstico, menor función física y resiliencia. Igualmente, la resiliencia mostró una correlación significativa y positiva con varias dimensiones de la calidad de vida, siendo las más robustas con vitalidad y con los índices sumarios de salud física y mental. 
Tabla 4. Análisis de correlación rho de Spearman entre edad, tiempo de diagnóstico, BIS, HAD y CD-RISC 10 con CVRS.

\begin{tabular}{|c|c|c|c|c|c|c|c|c|c|c|c|}
\hline & & $\begin{array}{l}\text { Función } \\
\text { física }\end{array}$ & $\begin{array}{c}\text { Rol } \\
\text { físico }\end{array}$ & $\begin{array}{c}\text { Dolor } \\
\text { corporal }\end{array}$ & $\begin{array}{c}\text { Salud } \\
\text { general }\end{array}$ & Vitalidad & $\begin{array}{c}\text { Función } \\
\text { social }\end{array}$ & $\begin{array}{c}\text { Rol } \\
\text { emocional }\end{array}$ & $\begin{array}{l}\text { Salud } \\
\text { mental }\end{array}$ & $\begin{array}{c}\text { Índice } \\
\text { sumario } \\
\text { de salud } \\
\text { física }\end{array}$ & $\begin{array}{c}\text { Índice } \\
\text { sumario } \\
\text { de salud } \\
\text { mental }\end{array}$ \\
\hline \multirow{7}{*}{ 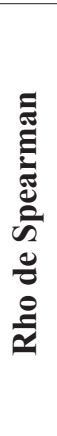 } & Edad & -.246 & .056 & $.281 *$ & .217 & .230 & .045 & .057 & .155 & .192 & .141 \\
\hline & $\begin{array}{l}\text { Tiempo de } \\
\text { diagnóstico }\end{array}$ & $-.451 * *$ & -.150 & -.105 & -.216 & $-.308^{*}$ & -.117 & -.145 & -.111 & $-.313^{*}$ & -.268 \\
\hline & $\begin{array}{l}\text { Total } \\
\text { CD-RISC } 10\end{array}$ & $.285^{*}$ & .232 & $.439 * *$ & $.343^{*}$ & $.516^{* *}$ & $.335^{*}$ & $.340^{*}$ & $.328 *$ & $.485^{* *}$ & $.475 * *$ \\
\hline & Total_HHI & .110 & .206 & $.380 * *$ & $.372 * *$ & $.354^{*}$ & $.394 * *$ & $.493 * *$ & $.388 * *$ & $.411 * *$ & $.535 * *$ \\
\hline & Total_BIS & .079 & -.238 & $-.345^{*}$ & $-.476 * *$ & $-.395 * *$ & -.267 & $-.293^{*}$ & $-.472 * *$ & $-.376 * *$ & $-.514 * *$ \\
\hline & HADS_ANS & .020 & -.210 & $-.374 * *$ & $-.523^{* *}$ & $-.286^{*}$ & -.245 & $-.509 * *$ & $-.658 * *$ & $-.365^{* *}$ & $-.559 * *$ \\
\hline & HADS_DEP & -.218 & $-.415 * *$ & $-.471 * *$ & $-.543 * *$ & $-.571 * *$ & $-.495 * *$ & $-.567 * *$ & $-.639 * *$ & $-.637 * *$ & $-.764 * *$ \\
\hline
\end{tabular}

* Correlación significativa al nivel 0.05 (bilateral).

** Correlación significativa al nivel 0.01 (bilateral).

La esperanza mostró correlaciones significativas positivas con las dimensiones de calidad de vida del MOS-SF 36, sobre todo con los índices sumarios de salud física y mental y el rol emocional, mientras que la imagen corporal las obtuvo también con el índice sumario de salud mental, salud general y salud mental.

Por último, tanto la ansiedad como la depresión correlacionaron significativamente en dirección negativa. La ansiedad se relacionó inversamente con salud mental, índice sumario de salud mental y salud general, mientras que la depresión lo hizo negativamente con todas las dimensiones, sobre todo con ambos índices sumarios y con salud general.

\section{DISCUSIÓN}

La presente investigación tuvo como propósito evaluar la relación de las variables salutogénicas (resiliencia y esperanza) y patogénicas (ansiedad y depresión), calidad de vida relacionada con la salud e imagen corporal en 50 pacientes diagnosticadas con cáncer de mama atendidas en el Instituto Médico de Alta Tecnología (IMAT) de Montería, Colombia. Con relación a la ansiedad y la depresión, en este estudio no se encontraron niveles clínicos de estas variables, según los puntos de corte para muestras de pacientes oncológicos colombianos hallados por Rico et al. (2005) en su validación del HADS en el país. Estos datos concuerdan con los de diferentes estudios hechos con enfermos crónicos colombianos, en los que se han observado niveles bajos de emociones negativas, como lo ilustra la investigación de Gaviria, Vinaccia, Riveros y Quiceno (2007), pero difieren de los de Fradelos et al. (2017), y de los datos colombianos de Finck y Forero (2011) y Moreno, Krikorian y Palacio (2015), quienes hallaron niveles de depresión y ansiedad clínicamente significativos en pacientes oncológicos. Sin embargo, cabe aclarar que en esa última investigación se incluyeron pacientes con otros tipos de cáncer, adicionales al de mama, así como varones, quienes obtuvieron porcentajes más elevados en depresión y más bajos en competencia en relación con las mujeres.

Otra variable a tomar en cuenta en esos estudios es el estadio de la enfermedad. Se ha establecido en la literatura relativa al cáncer y a los trastornos afectivos que en estadios más avanzados se agudizan la ansiedad y la depresión (cf. Fradelos et al., 2017); sin embargo, las participantes en el presente estudio se encontraban en su mayoría en el estadio II, lo que podría explicar sus bajos niveles en ambas condiciones.

Respecto a la imagen corporal, las medias de las pacientes fueron muy inferiores a las encontradas en pacientes con cáncer de mama por Hopwood et al. (2001), autores de la escala BIS, y asimismo por Gómez et al. (2015), quienes hicieron la validación española, lo que significa que estas pacientes tuvieron niveles óptimos en lo que a esta variable se refiere, no obstante los diversos procedimientos quirúrgicos a los que se habían sometido y sus probables afectaciones de todo tipo, 
y mantenían una buena percepción de sí mismas en cuanto a su imagen corporal. A este respecto, es importante recordar que $40 \%$ de las pacientes del presente estudio no se habían sometido a cirugías y que solo $22 \%$ de ellas habían sufrido una mastectomía radical, lo que puede haber favorecido dicha percepción positiva. Si bien Die-Trill y Die-Goyanes (2003) y Segura, García y Saúl (2014) señalan que las mujeres sometidas a cirugías conservadoras preservan mejor su imagen corporal que las que se someten a cirugías radicales, es importante señalar que el solo tratamiento sistemático con quimioterapia o radioterapia repercute también en la imagen corporal, la libido y la fertilidad de estas mujeres (Sebastián, 2003; Sebastián, Manos, Bueno y Mateos, 2007).

En cuanto a la variable de resiliencia medida con el cuestionario CD-RISC 10, la muestra alcanzó puntajes de medios a altos, al igual que en los estudios estadounidenses de validación del CD-RISC 10 en población general (Campbell-Sills y Stein, 2007) y en los hechos con pacientes con cáncer de mama (cf. Markovitz, Schrooten, Arntz y Peters, 2015; Scali et al., 2012).

En lo referente a la esperanza, los puntajes obtenidos en la presente investigación muestran niveles altos, según los puntos de corte hallados en el estudio de Heidari y Ghodusi (2015) en una muestra de cien mujeres iraníes con diagnóstico de cáncer de mama. La esperanza es esencial para los pacientes con cáncer en virtud de que los ayuda a lidiar con el diagnóstico y con los diferentes tratamientos (Balsanelli y Grossi, 2016). En el caso presente, los puntajes de las pacientes correlacionaron positivamente con las dimensiones de calidad de vida del MOS-SF 36, como vitalidad, rol emocional y los índices sumarios de salud física y mental. En un estudio con 213 mujeres chinas con cáncer de mama en que se utilizaron el CD-RISC 10 y el HHI como instrumentos para medir la resiliencia y la esperanza, respectivamente, se halló una correlación positiva entre ambas, siendo la última un factor predictor de la primera (Wu, Liu, Li y Li, 2016). En el presente estudio se encontraron las mismas relaciones positivas.

Finalmente, con relación a los resultados de CVRS medido con el MOS-SF 36, estos fueron de buenos a muy favorables en todas las dimensiones, así como en los índices sumarios de salud mental y de salud física, mismos que son semejantes a los observados en algunas investigaciones hechas con enfermos de cáncer de mama, como las llevadas a cabo en China por Xiao et al. (2016) y en Corea por Lee, Min, Park y Jung (2012), aunque difieren de los hallados por Aguirre et al. (2017) en su estudio hecho en Colombia, en el que los puntajes de calidad de vida se ubicaron por debajo de 50 en todas las dimensiones físicas y mentales.

Llama la atención que la única dimensión de calidad de vida que aquí alcanzó niveles bajos fue el rol físico, que es el grado en que la salud física interfiere con el trabajo y con otras actividades diarias, lo que implica un rendimiento menor al deseado. Este resultado coincide con lo hallado en Perú por Lostaunau, Torrejón y Cassaretto (2017) en 53 mujeres diagnosticadas con cáncer de mama, en las que también el rol físico obtuvo puntajes significativamente menores en el MOS-SF 36 que en las demás dimensiones, lo que podría deberse a los efectos del tratamiento oncológico.

En cuanto a las relaciones más significativas encontradas entre las variables estudiadas, deben apreciarse las positivas que hay entre la resiliencia y las dimensiones de vitalidad, dolor corporal y los índices sumarios de salud física y mental del MOS-SF 36. Numerosos estudios con pacientes de cáncer de mama han analizado el importante papel que tiene la resiliencia como factor protector ante el dolor, el cansancio y el agotamiento debidos a la propia enfermedad y a los tratamientos médico-quirúrgicos (Ristevska-Dimitrovska et al., 2015).

Las fuertes correlaciones negativas encontrada entre ansiedad y depresión y las diferentes dimensiones de la CVRS, como vitalidad, rol emocional e índices sumarios de salud física y mental, coinciden con las halladas en estudios hechos en otras partes de mundo, en los que se observó que la depresión se asocia con niveles altos de desesperanza y discapacidad en pacientes con cáncer de mama (Vin-Raviv, Akinyemiju, Galea y Bovbjerg, 2015; Zainal, Nik-Jaafar, Baharudin, Sabki y $\mathrm{Ng}, 2013)$.

Finalmente, ante las correlaciones positivas que hubo entre la esperanza y las dimensiones de rol emocional, índice sumario de salud mental y calidad de vida del MOS-SF 36, se plantea una relación entre dicha esperanza y el estado men- 
tal, ya vista en diferentes estudios sobre el cáncer de mama (Heidari y Ghodusi, 2015; Zhang, Gao, Wang y Wu, 2010).

En conclusión, es posible que los resultados del presente estudio sirvan de base para obtener una mayor información acerca de la relación de las citadas variables y la influencia que ejercen entre sí, de manera que puedan llevarse a cabo intervenciones mediante las cuales se brinden mejores herramientas de afrontamiento a quienes padecen esta enfermedad (Sánchez, Santaballa, Munárriz, Pérez y Montalar, 2009; Sebastián et al., 2007).
Dentro de las limitaciones del estudio es posible anotar que la escala BIS (Body Image Scale) está traducida pero no adaptada culturalmente a la población colombiana con cáncer. También es importante señalar que la cantidad de sujetos que participaron en la investigación fue reducida, y además que el diseño de la investigación permitió solamente un nivel correlacional, por lo que los resultados y las interpretaciones de los mismos deben limitarse a la población del IMAT investigada.

\section{REFERENCIAS}

Aguirre L., H., Núñez, C., Navarro, A. y Cortés, S. (2017). Calidad de vida según el estadio del cáncer de seno en mujeres: análisis desde el FACT-B y el SF-36. Psychología: Avances en la Disciplina, 11(1), 109-120.

Alonso, J., Regidor, E., Barrio, G., Prieto, L., Rodríguez, C. y De la Fuente, L. (1998). Valores poblacionales de referencia de la versión española del Cuestionario de Salud SF-36. Medicina Clínica, 111, 410-416.

Alonso, J., Prieto, L. y Antó, J. (1995). La versión española del SF-36 Health Survey (Cuestionario de Salud SF-36): un instrumento para la medida de los resultados clínicos. Medicina Clínica, 104, 771-776.

American Psychological Association (2017). Ethical principles of psychologists and code of amputation. Adapted Physical Activity Quarterly, 26, 236-258.

Angner, E., Ghandi, J., Williams-Purvis, K., Amante, D. y Allison, J. (2013). Daily functioning, health status and happiness in older adults. Journal of Happiness Study, 14, 1563-1574.

Angner, E., Ray, M., Saag, K. y Allison, J. (2009). Health and happiness among older adults. Journal of Health Psychology, 14(4), 503-512.

Balsanelli, A. y Grossi, S. (2016). Factores predictivos de esperanza entre mujeres con cáncer de mama durante el tratamiento quimioterapéutico. Revista de la Escuela Enfermería USP, 50(6), 898-904.

Campbell-Sills, L. y Stein, M.B. (2007). Psychometric analysis and refinement of the Connor-Davidson Resilience Scale (CD-RISC): Validation of a 10-item measure of resilience. Journal of Traumatic Stress, 20, 1019-1028.

Castilla, H., Urruti, C., Shimabukuro, M. y Caycho, T. (2014). Análisis psicométrico del índice de esperanza de Herth en una muestra no clínica peruana. Psicología desde el Caribe, 31(2), 187-206.

Die-Trill, M. y Die-Goyanes, A. (2003). Cirugía oncológica. En M. Die-Trill (Ed.): Psicooncología (pp. 263-274). Culla (Castelló, España): Hades Ediciones.

Eliott, J.A. y Oliver, I.N. (2009). Hope, life, and death: a qualitative analysis of dying. Cancer patients' talk about hope. Death Studies, 33, 609-638.

Finck, C. y Forero, M. (2011). Ansiedad y depresión en pacientes con cáncer de seno y su relación con la espiritualidad/religiosidad: análisis preliminar de datos. Revista de Psicología de la Universidad de Antioquia, 3(1), 7-27.

Fradelos, E., Papathanasiou, I., Veneti, A., Daglas, A., Christodoulou, E., Zyga, S. y Kourakos, M. (2017). Psychological distress and resilience in women diagnosed with breast cancer in Greece. Asian Pacific Journal of Cancer Prevention, 18(9), 2545-2550.

Fredrickson, B.L. (2001). The role of positive emotions in positive psychology. The broaden-and-build theory of positive emotions. The American Psychologist, 56(3), 218-226.

Garaigordobil, M. (2015). Predictor variables of happiness and its connection with risk and protective factors for health. Frontiers in Psychology, 6, 1176. doi: 10.3389/fpsyg.2015.01176.

Gaviria, A.M., Vinaccia, S., Riveros, M.F. y Quiceno, J.M. (2007). Calidad de vida relacionada con la salud, afrontamiento al estrés y emociones negativas en pacientes con cáncer en tratamiento quimioterapéutico. Psicología desde el Caribe, 20, 50-75.

Gómez, P., Bragado, C., Hernández, M. y Sánchez, M.L. (2015). The Spanish version of the Body Image Scale (S-BIS): psychometric properties in a sample of breast and gynecological cancer patients. Supportive Care in Cancer, 23(2), 473-481.

Halding, A. y Heggdal, K. (2012). Patients' experiences of health transitions in pulmonary rehabilitation. Nursering Inquires, 19, 345-356. 
Heidari, M. y Ghodusi, M. (2015). The relationship between body esteem and hope and mental health in breast cancer patients after mastectomy. Indian Journal of Palliative Care, 21(2), 198-202. doi: 10.4103/0973-1075.156500.

Herth, K. (1991). Development and refinement of an instrument to measure hope. Scholarly Inquiry for Nursing Practice, 5(1), $39-51$.

Hopwood, P., Fletcher, I., Lee, A. y Al Ghazal, S. (2001). A body image scale for use with cancer patients. European Journal of Cancer, 37(2), 189-197.

Horgan, O. y MacLachlan, M. (2004). Psychosocial adjustment to lower-limb amputation: a review. Disability Rehabilitation, 26(8), 37-50.

International Union of Psychological Science (2008). Declaración Universal de Principios Éticos para Psicólogos. Disponible en https://www.ucc.ie/en/media/academic/appliedpsychology/ethicsforms/universal_declaration_of_ethics.pdf.

Lee, S.H., Min, Y.S., Park, H.Y. y Jung, T.D. (2012). Health-related quality of life in breast cancer patients with lymphedema who survived more than one year after surgery. Journal of Breast Cancer, 15(4), 449-453.

Lostaunau, V., Torrejón, C. y Cassaretto, M. (2017). Estrés, afrontamiento y calidad de vida relacionada a la salud en mujeres con cáncer de mama. Actualidades en Psicología, 31(122). doi: http://dx.doi.org/10.15517/ap.v31i122.25345.

Lugo, L., García, H. y Gómez, C. (2006). Confiabilidad del cuestionario de calidad de vida en salud SF-36 en Medellín, Colombia. Revista de la Facultad Nacional de Salud Pública, 24(2), 37-50.

Markovitz, S., Schrooten, W., Arntz A. y Peters, M.L. (2015). Resilience as a predictor for emotional response to the diagnosis and surgery in breast cancer patients. Psychooncology, 24, 1639-1645. doi: 10.1002/pon.3834.

Martínez, S. (2018). Calidad de vida y apoyo social en mujeres con cáncer de mama. Ciencia y Cuidado, 15(1). Recuperado de http://revistas.ufps.edu.co/ojs/index.php/cienciaycuidado/article/view/1232/1270.

Ministerio de Salud y Protección Social (2017a). ABC del cáncer en Colombia. Generalidades. ¿Cuáles son los principales tipos de cáncer más comunes en la población colombiana? Disponible en https://www.minsalud.gov.co/salud/publica/PENT/ Paginas /Prevenciondel-cancer.aspx.

Ministerio de Salud y Protección Social (2017b). Boletín de prensa 298 de 2014: Cáncer de mama, una enfermedad en ascenso en Colombia. Disponible en https://www.minsalud.gov.co/Paginas/-Cancer-de-mama,-una-enfermedad-en-ascenso-en-Colombia.aspx.

Montalvo, A., Fajardo, H., Angulo, T., Flórez, D., Caffroni, R. y Fajardo, Y. (2016). Condiciones sociodemográficas y nivel de incertidumbre en mujeres ante el diagnóstico de cáncer de mama. Hacia la Promoción de la Salud, 21(2), 114-126.

Moreno, A., Krikorian, A. y Palacio, C. (2015). Malestar emocional, ansiedad y depresión en pacientes oncológicos colombianos y su relación con la competencia percibida. Avances en Psicología Latinoamericana, 33(3), 517-529.

Notario, B., Soler, M., Serrano, M.D., Bartolomé, R., García C., J. y Martínez, V. (2011). Reliability and validity of the Spanish version of the 10-item Connor-Davidson Resilience Scale (10-item CD-RISC) in young adults. Health and Quality of Life Outcomes, 9, 63. doi:10.1186/1477-7525-9-63.

Ong, A., Zautra, A. y Reid, M.C. (2010). Psychological resilience predicts decreases in pain catastrophizing through positive emotions. Psychology and Aging, 25(3), 516-523.

Organización Mundial de la Salud (2018). Cáncer. Datos y cifras. Disponible en http://www.who.int/es/news-room/fact-sheets / detail/cancer.

Organización Panamericana de la Salud (2018). Cáncer de mama. Disponible en: http://www.paho.org/hq/index.php?option = com_contenty view $=$ articley id $=5041 \% 3 \mathrm{~A} 2011$-breast-cancery catid $=1872 \% 3$ Acancery Itemid $=3639 \mathrm{y}$ lang $=$ es.

Pineda, S., Andrade, S. y Montoya, Y. (2013). Factores asociados a la calidad de vida con cáncer de mama. Gerencia en Políticas de Salud, 16(32), 85-95.

Reich, M., Lesur, A. y Pedrizet, C. (2008). Depression, quality of life and breast cancer: a review of literature. Breast Cancer Research and Treatment, 110(1), 9-17.

Richman, L.S., Kubzansky, L., Maselko, J., Kawachi, I., Choo, P. y Bauer, M. (2005). Positive emotion and health: going beyond the negative. Health Psychology, 24, 422-429.

Rico, J., Restrepo, M. y Molina, M. (2005). Adaptación y validación de la escala hospitalaria de ansiedad y depresión (HADS) en una muestra de pacientes con cáncer del Instituto Nacional de Cancerología de Colombia. Revista del Laboratorio de Psicometría, 3, 73-86.

Rincón F., M.E., Pérez S.G., M.A., Borda M., M. y Rodríguez, A.M. (2012). Impacto de la reconstrucción mamaria sobre la autoestima y la imagen corporal en pacientes con cáncer de mama. Universitas Psychologica, 11(1), 25-41.

Ristevska-Dimitrovska, G., Stefanovski, P., Smichkoska, S., Raleva, M. y Dejanova, B. (2015). Depression and resilience in breast cancer patients. Open Access Macedonian Journal of Medical Sciences, 3(4), 661-665.

Riveros, F., Bernal, L., Bohórquez, D., Vinaccia, S. y Quiceno, J. (2016). Validez de constructo y confiabilidad de la Connor-Davidson Resilience Scale (CD-RISC 10) en población colombiana con enfermedades crónicas. Salud y Sociedad, 7(2), $130-137$. 
Salas, C. y Grisales, H. (2010). Calidad de vida y factores asociados en mujeres con cáncer de mama en Antioquia, Colombia. Revista Panamericana de Salud Pública, 28(1), 9-18.

Sánchez, J., Santaballa, A., Munárriz, B., Pérez, J. y Montalar J. (2009). Mujeres con cáncer de mama: evaluación del afecto positivo y negativo y valoración de un programa de intervención psicológica en el ámbito hospitalario. Psicooncología, $6(1), 139-154$.

Scali, J., Gandubert, C., Ritchie, K., Soulier, M., Ancelin, M. y Chaudieu, I. (2012). Measuring resilience in adult women using the 10-items Connor-Davidson Resilience Scale (CD-RISC). Role of trauma exposure and anxiety disorders. PLOS One, 7, e39879.

Sebastián, J. (2003). Repercusiones psicosociales de los tratamientos oncológicos. En V. Valentin (Ed.): Oncología y atención primaria (pp. 785-792). Madrid: Novartis.

Sebastián, J., Manos, D., Bueno, N. y Mateos, M. (2007). Imagen corporal y autoestima en mujeres con cáncer de mama participantes en un programa de intervención psicosocial. Clínica y Salud, 18(2), 137-161.

Segura, M., García, R. y Saúl, L. (2014). Imagen corporal y autoestima en mujeres mastectomizadas. Psicoocología, 11(1), $45-57$.

Seligman, M.E.P. y Csikszentmihalyi, M. (2000). Positive psychology: An introduction. American Psychologist, 55(1), 5-14.

Sousa, A.I., Corredeira, R. y Pereira, A.L. (2009). The body in persons with an amputation. Adapted Physical Activity Quarterly, 26(3), 236-258.

Taub, D., Blinde, E. y Greer, K. (1999). Stigma management through participation in sport and physical activity: Experiences of male college students with physical disabilities. Human Relations, 52(11), 1469-1484.

Tugade, M., Fredrickson, B. y Feldman, L. (2004). Psychological resilience and positive emotional granularity: Examining the benefits of positive emotions on coping and health. Health Journal of Personality, 72(6), 1161-1190.

Vin-Raviv, N., Akinyemiju, T., Galea, S. y Bovbjerg, D. (2015). Depression and anxiety disorders among hospitalized woman with breast cáncer. PLOS One, 10(6), e0129169.

Ware, J.E. y Sherbourne, C.D. (1992). The MOS 36-Item Short-Form Health Survey (SF-36): I. conceptual framework and item selection. Medical Care, 30(6), 473-83.

Waynor, W., Gao, N., Dolce, J., Haytas, L. y Reilly, A. (2012). The relationship between hope and symptoms. Journal of Psychiatry and Rehabilitation, 35, 345-348.

World Medical Organization (2013). Declaración de Helsinki de la AMM: Principios éticos para las investigaciones médicas en seres humanos. Disponible en http://www.isciii.es/ISCIII/es/contenidos/fd-investigacion/fd-evaluacion/fd-evaluacion-etica-investigacion/Declaracion-Helsinki-2013-Esp.pdf.

Wu, Z., Liu, Y., Li, X. y Li, X. (2016). Resilience and associated factors among mainland Chinese women newly diagnosed with breast cancer, PLOS One, 11(12), e0167976. doi: 10.1371 / journal.pone.0167976.

Xiao, C., Miller, A., Felger, J., Mister, B., Liu, T., y Torres, M. (2016). A prospective study of quality of life in breast cancer patients undergoing radiation therapy. Advances in Radiation Oncology, 1, 10-16.

Zainal, N.Z., Nik-Jaafar, N.R., Baharudin, A., Sabki, Z.A. y Ng, C.G. (2013). Prevalence of depression in breast cancer survivors: a systematic review of observational studies. Asian Pacific Journal of Cancer Prevention, 14(4), $2649-2656$.

Zhang, H., Zhao, Q., Cao, P. y Ren, G. (2017). Resilience and quality of life: Exploring the mediator role of social support in patients with breast cancer. Medicine Science Monitor, 23, 5969-5979.

Zhang, J., Gao, W., Wang, P. y Wu, Z.H. (2010) Relationships among hope, coping style and social support for breast cancer patients. Chinese Medical Journal, 123(17), 2331-2335.

Zigmond, A.S. y Snaith, R.P. (1983). The hospital anxiety and depression scale. Acta Psychiatrica Scandinavica, 67(6), 361-370. 